\title{
Faciohypoglossal Anastomosis:
}

\section{Does the Morphology of the Facial Nerve Affect the Functional Result?}

\begin{abstract}
We report the results of a histological study of facial nerve specimens obtained at the time of faciohypoglossal anastomosis. We examined sections from 24 patients using light and electron microscopy in order to determine the extent of the degenerative process with time and its relationship to the functional results of the anastomosis. We graded the histological features associated with degeneration, regeneration, and fibrosis on an ordered nominal scale from 0 to 3 and correlated them with duration of facial palsy and postoperative facial function. There was no histological evidence of increasing collagenosis or fibrosis with duration of facial palsy, and endoneural fibrosis was a very infrequent finding. There was no evidence that the relative numbers of endoneural fibrocytes or the degree of collagenosis had any bearing on the functional result. The presence of large numbers of endoneural tubes, containing nonmyelinated axons, showed a positive correlation with the postoperative facial function, whereas the presence of active axon degeneration showed a negative correlation. (Skull Base Surgery, 4(4): 193-201, 1994)
\end{abstract}

Faciohypoglossal anastomosis is currently the procedure of choice for restoration of facial function when direct anastomosis or cable grafting is not possible. The functional results of grafting may be influenced by the age of the patient and the nature of the original injury, both of which are outside the influence of the surgeon. The timing of the repair, however, is to some degree controllable, and it remains unclear how long after injury the repair should be carried out. There are compelling histological arguments in favor of early repair. Following peripheral nerve injury in experimental animals the myelin sheaths degenerate and are phagocytosed, this process being well advanced by 36 to 48 hours. ${ }^{1}$ The axons break up after 48 to 72 hours and usually disappear after 2 weeks. Schwann cells replicate and align themselves along the empty axon tubes to form endoneural tubes or "bands of Büngner." Following these changes there is an increase in endoneural collagen and the tubes shrink progressively so that after the first 3 months they have lost approximately $80 \%$ to $90 \%$ of their original diameter. If they are not reinnervated they will ultimately atrophy. ${ }^{1}$ This suggests that the sooner repair is carried out, the greater the likelihood that ingrowing axons will prevent the shrinking of the axon tubes. ${ }^{2}$ However, these experimental changes may not be comparable with the situation in the human facial nerve. A study of the histology of seven patients with facial palsy after acoustic tumor removal found that the degree of collagenization appeared unrelated to the time lapse since injury but was related to the number of fibers in the motor areas. ${ }^{3}$ Similarly, histological examination of the distal stumps of five transected facial nerves obtained at faciohypoglossal reinnervation surgery suggests that the human facial nerve may undergo less denervation atrophy that has been demonstrated in the peripheral nerves of experimental animals. ${ }^{4}$

The results of clinical studies conflict; in a review of 40 cases, Gavron and Clemis concluded that the best results were obtained in those cases in which the anastomosis was carried out early and that a delay of more than one year adversely affected the prognosis. ${ }^{5}$ However, this experience is not universal; a delay of 12 to 15 months did

Skull Base Surgery, Volume 4, Number 4, October 1994 ENT Department, Kent and Sussex Hospital, Mount Ephraim, Tunbridge Wells, Kent, England (G.B.) and ENT Department, University Hospital, Zurich, Switzerland (H.F., U.F) Reprint requests: Dr. Felix, ENT Department, University Hospital, 8091 Zurich, Switzerland Copyright (C) 1994 by Thieme Medical Publishers, Inc., 381 Park Avenue South, New York, NY 10016. All rights reserved. 
not adversely affect the outcome in one study, ${ }^{6}$ and good results have been obtained with anastomosis up to 4 years after the injury. ${ }^{7}$ We have examined facial nerve specimens obtained at the time of faciohypoglossal anastomosis in 24 patients in an attempt to answer two questions:

1. What is the extent of the degenerative process with time in the human facial nerve?

2. Do the histological changes relate to the functional results of the anastomosis?

\section{METHODS}

\section{Selection Criteria for Faciohypoglossal Anastomosis}

Faciohypoglossal anastomosis was used when the proximal stump of the facial nerve was not available or unsuitable for grafting. All cases had some preoperative facial tone, ie, at least $30 \%$ symmetry assessed at rest (Table 1 ).

\section{Technique}

The nerves were exposed through a modified parotidectomy incision (or extension of existing retroauricular incision) and the hypoglossal nerve sectioned before the first branch to the tongue. Central branches of the facial nerve were ligated in an attempt to direct fibers to the upper and lower parts of the face. The epineurium of both

Table 1. Detailed Evaluation of Facial Symmetry

\begin{tabular}{lrc}
\hline Facial Symmetry & $\%$ & Points \\
\hline At rest & 0 & 0 \\
& 30 & 6 \\
& 70 & 14 \\
Wrinkling forehead & 100 & 20 \\
& 0 & 0 \\
Closing eyes & 30 & 3 \\
& 70 & 7 \\
& 100 & 10 \\
Smiling & 0 & 0 \\
& 30 & 9 \\
& 70 & 21 \\
Whistling & 100 & 30 \\
& 0 & 0 \\
& 30 & 9 \\
& 70 & 21 \\
& 100 & 30 \\
& 0 & 0 \\
& 30 & 3 \\
& 70 & 70 \\
\hline
\end{tabular}

Total points used to express facial function as a percentage. $0 \%=$ complete asymmetry/complete absence of voluntary facial movement, $30 \%=$ poor symmetry (return of function closer to complete asymmetry than to normal symmetry of the face), $70 \%=$ fair symmetry (incomplete return of function closer to complete symmetry nerves was removed for $3 \mathrm{~mm}$ and the end of the facial nerve was split with neurectomy scissors to increase the anastomotic surface. Anastomosis was carried out using three or four 10-0 nylon sutures. The anastomosis was wrapped in a Cargyle membrane and stabilized with fibrin glue.

\section{Patient Follow-up and Pre- and Postoperative Facial Grading}

Functional results were recorded using the Fisch grading system 8 (Table 1). Follow-up was between 1 and 8 years $($ mean $=5, S D=2$ ). Postoperative results were unavailable in four cases.

\section{Histology}

Facial nerve biopsies were immediately immersionfixed in cold phosphate-buffered 3\% glutaraldehyde and postfixed with $1 \%$ osmium tetroxide. Following alcohol dehydration, the sections were embedded in epoxy resin. Semithin sections were stained with toluidine blue $O$ for light microscopy, and thin sections were stained with uranyl acetate and lead citrate for electron microscopy. Hypoglossal nerve sections were prepared for light microscopy in the same way.

\section{Statistics}

The difference between pre- and postoperative facial function was evaluated using Wilcoxon's signed rank test. The significance of the interrelationship between the histological features was assessed by reduction to $2 \times 2$ tables and applying Fisher's exact test. The interrelationship between postoperative facial function, age of the patients, and duration of palsy was tested using Kendall's rank correlation. The correlation between the duration of palsy and the histological features and the postoperative facial function and the histological features was assessed using Kendall's rank correlation.

\section{RESULTS}

\section{Age Distribution and Diagnosis}

The ages ranged from 18 months to 75 years $(47 \pm$ 18). Patient diagnoses are summarized in Table 2.

\section{Site of Lesion}

The site of the lesion was pregeniculate in 14 , involved the geniculate ganglion in 3 , was postgeniculate in 4 , and was unknown in 3 (Table 2). 
Table 2. Diagnostic Details

\begin{tabular}{|c|c|c|c|c|c|c|}
\hline \multirow{2}{*}{$\begin{array}{l}\text { Patient } \\
\text { Number }\end{array}$} & \multirow[b]{2}{*}{ Diagnosis } & \multirow[b]{2}{*}{ Age $(y)$} & \multirow[b]{2}{*}{ Duration of Palsy (mo) } & \multirow[b]{2}{*}{ Site of Lesion } & \multicolumn{2}{|c|}{ Facial Function } \\
\hline & & & & & Pre & Post (y) \\
\hline 1 & Acoustic neuroma & 48 & Per-op & IAM & 100 & $60(5)$ \\
\hline 2 & Acoustic neuroma & 39 & Per-op & IAM & 100 & $60(8)$ \\
\hline 3 & Mastoidectomy & 28 & 1 & $T$ & 9 & - \\
\hline 4 & Acoustic neuroma & 68 & 3 & IAM & 6 & $60(5)$ \\
\hline 5 & Glomus tumor & 33 & 4 & L-M & 62 & $55(1)$ \\
\hline 6 & Glomus tumor & 43 & 6 & G-T & 30 & $50(8)$ \\
\hline 7 & Acoustic neuroma & 55 & 7 & IAM & 6 & $69(5)$ \\
\hline 8 & Meningioma & 47 & 11 & IAM & 23 & $35(1)$ \\
\hline 9 & Acoustic neuroma & 54 & 11 & IAM & 6 & 60 (7) \\
\hline 10 & Acoustic neuroma & 68 & 16 & IAM & 6 & - \\
\hline 11 & Cholesteatoma & 68 & 16 & IAM-L & 26 & $53(5)$ \\
\hline 12 & Congenital & 1.5 & 18 & Unknown & 6 & $57(5)$ \\
\hline 13 & Idiopathic & 75 & 24 & Unknown & 6 & $27(2)$ \\
\hline 14 & Ceruminous adenoma & 60 & 24 & T-M & 38 & - \\
\hline 15 & Glomus tumor & 29 & 24 & $M$ & 6 & $59(5)$ \\
\hline 16 & Idiopathic & 52 & 24 & CPA & 6 & $50(4)$ \\
\hline 17 & Longitudinal fracture & 23 & 28 & $\mathrm{~L}$ & 6 & $60(7)$ \\
\hline 18 & Cerebellar angioma & 17 & 28 & CPA & 6 & $60(5)$ \\
\hline 19 & Cholesteatoma & 46 & 32 & L & 30 & $72(5)$ \\
\hline 20 & Cholesteatoma & 54 & 36 & $T-M$ & 6 & $50(3)$ \\
\hline 21 & Hemangioma & 59 & 60 & Unknown & 34 & - \\
\hline 22 & Acoustic neuroma & 48 & 64 & IAM & 9 & $60(5)$ \\
\hline 23 & Acoustic neuroma & 54 & 72 & IAM & 6 & $58(5)$ \\
\hline 24 & Glomus tumor & 48 & 84 & $G \pm$ other & 53 & $60(6)$ \\
\hline
\end{tabular}

$\mathrm{IAM}=$ internal meatus, $\mathrm{T}=$ tympanic, $\mathrm{L}=$ labyrinthine, $\mathrm{M}=$ mastoid, $\mathrm{G}=$ geniculate ganglion, $\mathrm{CPA}=$ cerebellopontine angle.

\section{Duration of Palsy}

The duration of palsy prior to anastomosis ranged from 0 ( 2 peroperative cases) to 7 years (mean $25 \pm 23$ months).

\section{Functional Results}

The preoperative facial function ranged from $6 \%$ to $100 \%(24 \pm 28 \%)$. The postoperative function ranged from $27 \%$ to $72 \%(56 \pm 10 \%)$. The modal postoperative function was $60 \%$. Postoperative function was significantly better than preoperative function $(P=.001)$. The postoperative function was not significantly related to the duration of the palsy, the age of the patients, or preoperative facial function. Similarly, there was no significant difference in postoperative function between neoplastic and non-neoplastic cases or between cases where the site of lesion was pregeniculate and those where the lesion was geniculate or postgeniculate (Mann-Whitney test).

\section{Histology}

\section{Hypoglossal Nerve}

All samples were histologically normal with the exception of patient number 8 , in whom the nerve showed widespread degeneration. Although there was no preoperative palsy and the nerve appeared normal at opera- tion, it is likely that the nerve was infiltrated by meningioma. Nerve fiber counts from two randomly selected nerves were 7194 and 8369.

\section{Facial Nerve}

The histological features are analyzed under the following headings. To compare the histological features and to analyze the relationship between them they were graded on an ordered nominal scale from 0 to 3 (maximal presence).

Degenerative Changes. These were in the presence and condition of the sensory and motor bundles of the nerve $(1=$ extensive degeneration, few surviving fibers; 2 $=$ moderate degeneration, clearly recognizable; $3=$ near normal, slight degeneration permissible) and in the presence of active axon degeneration with splitting and breakdown of the myelin sheaths and the presence of phagocytosis by macrophages or Schwann cells $(1=$ scanty/focal, 2 = moderate/generalized, $3=$ extensive/generalized).

Regenerative Changes. Two features were taken as indicative of regenerative activity. The presence of endoneural tubes and myelinated axons. Myelinated axons were considered to be regenerating if they were of small diameter and clustered in groups around Schwann cells ( $1=<100$ groups of fibers in section, $2=100$ to 400 , $3=>400$ ).

Fibrotic Changes. An increase in endoneural collagen and an increase in the numbers of fibrocytes within the nerve were considered as fibrotic changes $(1=$ mild changes; 2 = increase above the "normal" level in 
the degenerated facial nerve; $3=$ severe, amounting to light microscopic appearance of fibrosis). The presence of epi- and perineural fibrous thickening was also noted, although it was not graded because many of the sections were around the stylomastoid foramen.

\section{Peroperative Injury}

In two patients with large acoustic neuromas the nerves were attenuated and it was not possible to remove the tumor without sacrifice of the nerve. Histological sections of these nerves were normal except for dilatation of the axons in sections proximal to the tumor (axon block).

\section{Preoperative Injury}

\section{Extent of Degeneration}

Sections proximal to the stylomastoid foramen were available in 6 of the 14 nerves where the lesion was proximal to the geniculate ganglion. A well-formed sensory bundle was present in all sections (Fig. 1). The geniculate ganglion was involved in 3 cases. A partly degenerated sensory bundle was present in one nerve. This case was a glomus tumor that had infiltrated the geniculate ganglion but had not completely destroyed it. Preoperative facial function was 53\%, and histologically, the geniculate ganglion showed some intact ganglion cells. The lesion was postgeniculate in 3 cases. A sensory bundle was present in 2 of the nerves. In one of these the lesion was in the distal tympanic and mastoid segments and the sensory bundle was present in sections between the lesion and the geniculate ganglion. The second case was a glomus tumor that had invaded the mastoid portion of the nerve. A small bundle of about 50 fibers of mixed diameters was present in distal mastoid sections. The origin of these fibers is unclear. Degeneration of the motor part of the nerve was complete in 8 of the 22 patients. There was evidence of active axonal degeneration in 10 patients (Fig. 2). This was seen up to 72 months after the onset of the palsy. Similarly, evidence of phagocytosis was seen in 9 patients (Fig. 3) and was evident up to 36 months after the palsy.

\section{Extent of Regeneration}

Endoneural tube formation was evident to some degree in all of the 22 cases (Table 3 and Fig. 4). This was up to 7 years after the onset of the facial paralysis. In all cases, most of the endoneural tubes contained fine nonmyelinated axons. This was also true in one patient (number 3 ) in whom the facial nerve had been sectioned during cholesteatoma removal one month before. In some instances small-diameter myelinated axons were seen, and in some a small proportion of the endoneural tubes were empty. Small groups of regenerated fine myelinated axons were seen in all but one case. In the majority these were extremely sparse, but marked evidence of regeneration of myelinated axons was seen in 7 cases (Fig. 5).

\section{Collagenosis and Fibrosis}

An increase in endoneural collagen deposition was seen in all the cases of more than one month duration. This was mild in 10 cases, moderate in 10 , and pronounced in 1 case (Fig. 6). A small number of fibrocytes were evident in all cases; 3 cases showed a moderate increase, and in 3 there were large numbers. The light microscopic appearances of fibrosis were only seen in 1 case (Fig. 6). Electron microscopy showed that, despite the densely packed col-

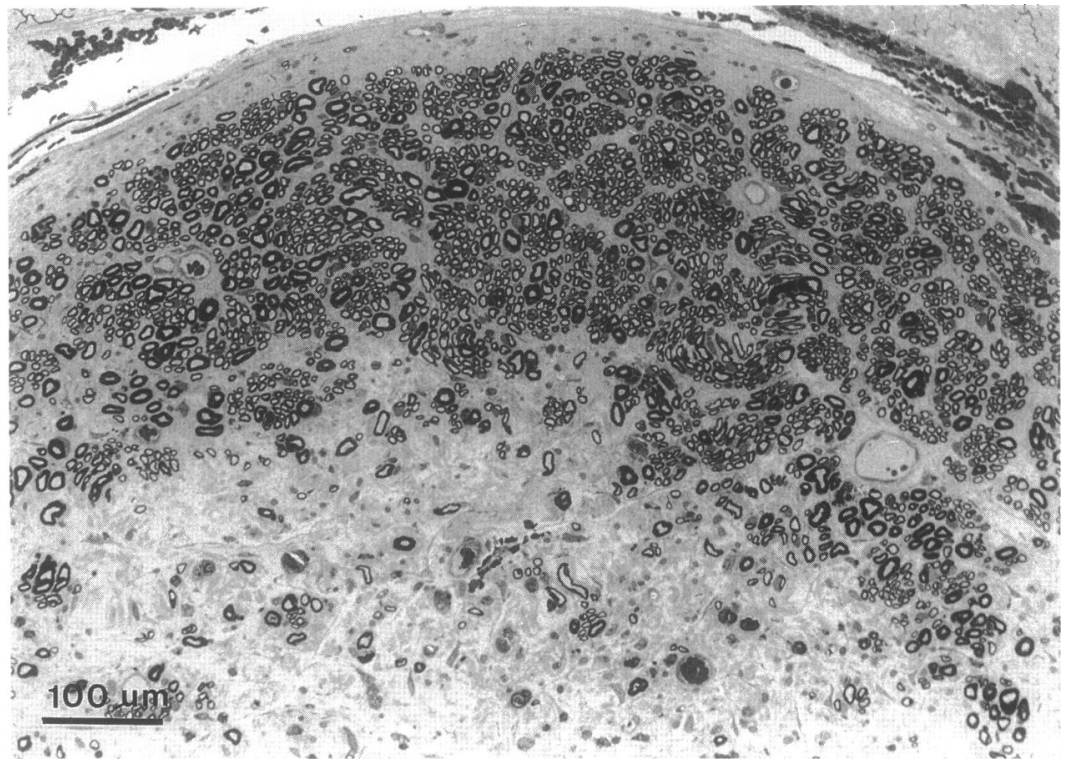

Figure 1. Transverse section through the proximal mastoid segment of case 4 showing a well-formed sensory bundle. Note the bimodal distribution of myelinated axons within the bundle. (Toluidine blue $\times 129$.) 
Figure 2. Distal mastoid segment of case 20 showing active degeneration of myelinated axons. (Toluidine blue $\times 408$.)

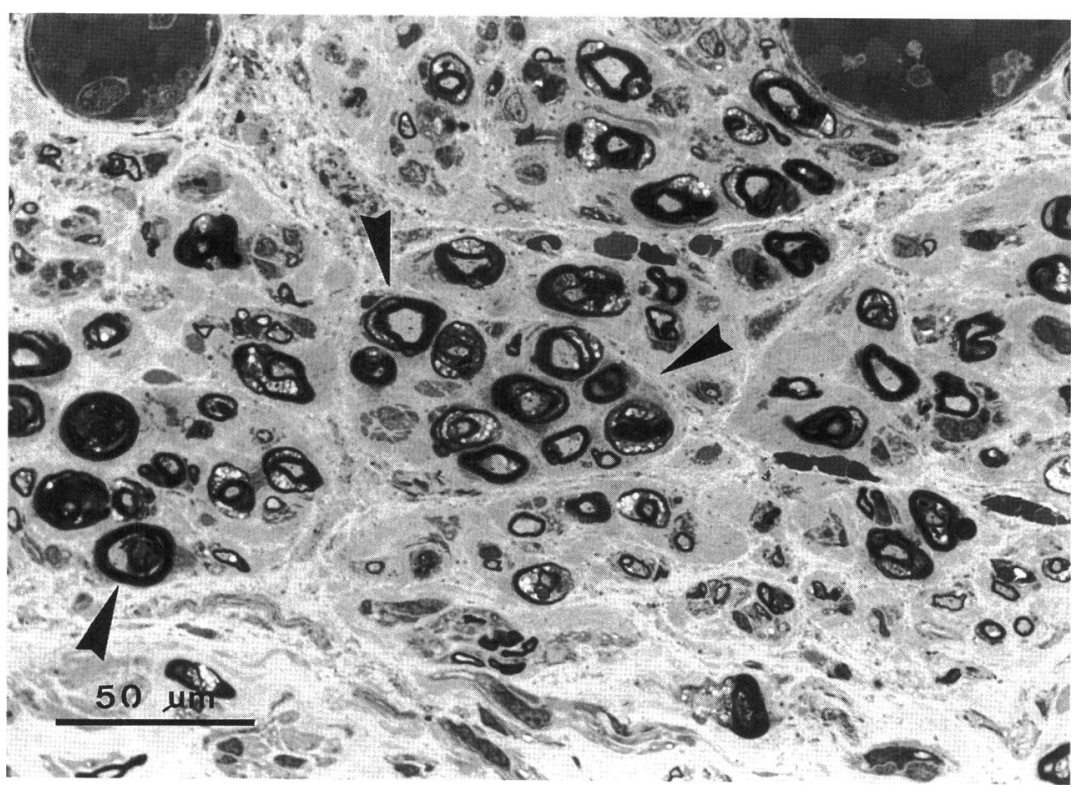

lagen, there were numerous endoneural tubes containing nonmyelinated axons (Fig. 7). An increase in fibrous tissue in the epi- and perineurium was seen in 14 patients. In 2 of the cases the coincidence of increased vascularity and perineural thickening produced a whorled appearance.

\section{Interrelationship between Histological Features}

There was a possible negative relationship between the relative number of fibrocytes and the presence of axon degeneration $(P=.04)$, between endoneural tubes and phagocytosis $(P=.02)$, and between myelinated axons and collagenosis $(P=.03)$. None of the other possible associations reached statistical significance.

\section{Correlation of Histological Features with Duration of Facial Palsy}

There was a positive correlation between duration and the relative numbers of endoneural tubes $(P=.05)$ and regenerated myelinated axons $(P=.03)$ and a negative correlation with the relative numbers of phagocytes $(P=.002)$.
Figure 3. Distal mastoid segment of case 3 . The nerve had been divided 1 month before. There is extensive phagocytosis. Many of the Schwann cells contain lipid globules from ingested myelin. (Toluidine blue $\times 504$.)

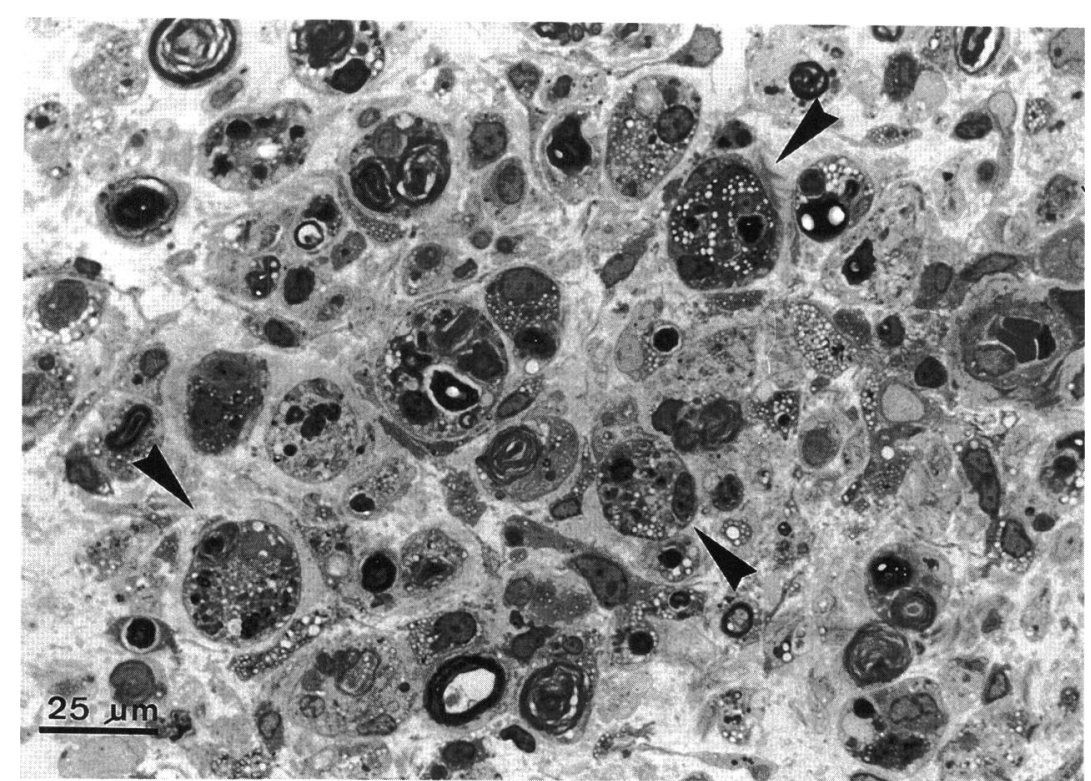


Table 3. Light and Electron Microscopy of Distal Facial Nerve Segment

\begin{tabular}{|c|c|c|c|c|c|c|}
\hline \multirow{2}{*}{$\begin{array}{l}\text { Patient } \\
\text { Number }\end{array}$} & \multicolumn{2}{|c|}{ Degeneration } & \multicolumn{2}{|c|}{ Regeneration } & \multicolumn{2}{|c|}{ Fibrosis } \\
\hline & Axons & Phagocytosis & ENT & $M A$ & Collagenosis & Fibrocytes \\
\hline 1 & 0 & 0 & 0 & 0 & 0 & 0 \\
\hline 2 & 0 & 0 & 0 & 0 & 0 & 0 \\
\hline 3 & 0 & 3 & 1 & 0 & 0 & 1 \\
\hline 4 & 1 & 2 & 1 & 1 & 2 & 3 \\
\hline 5 & 1 & 1 & 1 & 3 & 1 & 2 \\
\hline 6 & 2 & 2 & 1 & 1 & 1 & 2 \\
\hline 7 & 0 & 1 & 3 & 1 & 2 & 1 \\
\hline 8 & 2 & 1 & 1 & 1 & 1 & 1 \\
\hline 9 & 0 & 0 & 3 & 1 & 2 & 1 \\
\hline 10 & 2 & 1 & 2 & 1 & 2 & 1 \\
\hline 11 & 1 & 0 & 2 & 1 & 1 & 2 \\
\hline 12 & 0 & 0 & 3 & 2 & 1 & 1 \\
\hline 13 & 1 & 0 & 2 & 1 & 2 & 1 \\
\hline 14 & 0 & 0 & 1 & 3 & 1 & 1 \\
\hline 15 & 0 & 0 & 2 & 1 & 2 & 1 \\
\hline 16 & 0 & 0 & 1 & 1 & 3 & 3 \\
\hline 17 & 0 & 1 & 3 & 1 & 2 & 1 \\
\hline 18 & 0 & 0 & 2 & 2 & 1 & 1 \\
\hline 19 & 0 & 0 & 2 & 1 & 1 & 1 \\
\hline 20 & 3 & 2 & 1 & 1 & 2 & 1 \\
\hline 21 & 0 & 0 & 3 & 2 & 1 & 1 \\
\hline 22 & 1 & 0 & 3 & 2 & 2 & 1 \\
\hline 23 & 1 & 0 & 2 & 1 & 2 & 3 \\
\hline 24 & 0 & 0 & 2 & 3 & $\overline{1}$ & 1 \\
\hline
\end{tabular}

ENT = endoneural tubes; $\mathrm{MA}=$ myelinated axons.

\section{Correlation of Histological Features} with Postoperative Functional Results

There was a positive correlation between the postoperative result and the relative numbers of endoneural tubes $(P=.01)$ and a negative correlation with axon degeneration $(P=.004)$. There was no significant correlation with any of the other histological features. The mean postoperative result in the absence of axon degeneration was $61 \%$, and in the presence of axon degeneration it was
$50 \%$. The difference between the two groups was significant (Mann-Whitney test, $P=.02$ ).

\section{DISCUSSION}

The functional results were for the most part remarkably consistent, with a mean postoperative function of $56 \%$ and a modal value of $60 \%$. This supports the

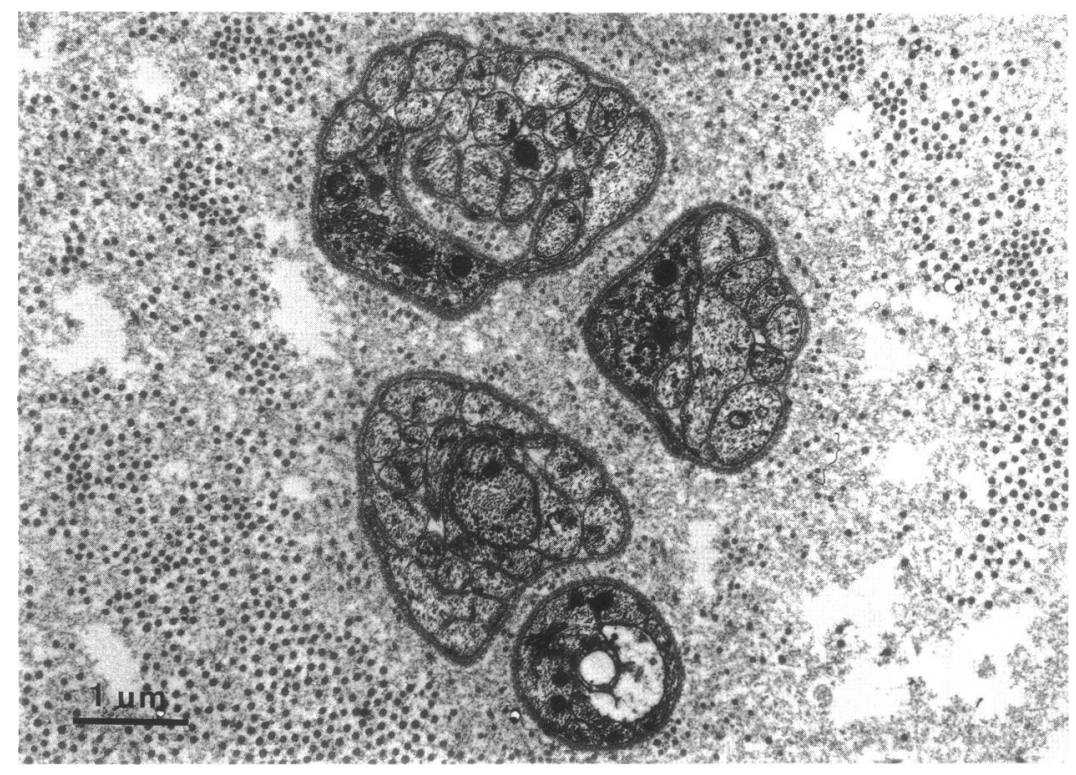

Figure 4. Electron micrograph of case 19. There are several endoneural tubes within the collagen fibrils. The Schwann cell cytoplasm is folded around nonmyelinated axons. (Uranyl acetate and lead citrate $\times 11400$.) 
Figure 5. Transverse section through the extratemporal part of the nerve of case 5 . Numerous regenerated myelinated axons are seen in clusters around Schwann cells. (Toluidine blue $\times 510$.)

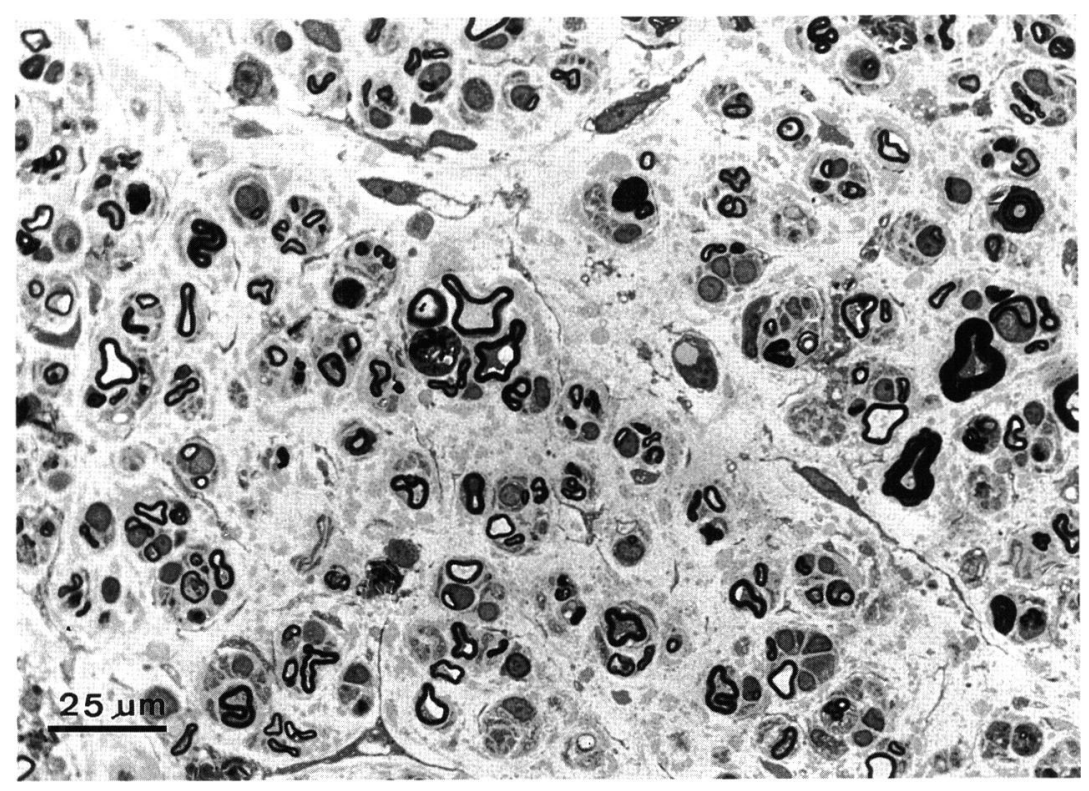

widespread opinion that faciohypoglossal anastomosis is the workhorse operation in this situation.

There were two noticeably poor results. One patient had a return of function of $35 \%$, which is most probably accounted for by the histological features of degeneration within the hypoglossal nerve. This was not clinically detected before surgery, and thus we emphasize the need for frozen section confirmation of the state of the donor nerve at the time of operation. A second patient had a return of function of $27 \%$. The patient was elderly (75) and had an idiopathic palsy that did not fit into the pattern of a Bell's palsy. It was thought that this may have been central in origin. There were certainly no distinguishing histological features of the facial nerve in this case.
The two cases in which the nerve was severed peroperatively had $100 \%$ preoperative facial function and facial nerves that might be regarded as histologically optimal for anastomosis. It is interesting that despite this the return of facial function ( $60 \%$ in both cases) was the modal value and little better than the mean.

It is a widely held view that the duration of the facial palsy at the time of anastomosis is a critical factor in determining the outcome and that poor results are to be anticipated after 18 months to 2 years. ${ }^{2}$ In their review of 40 patients, Gavron and Clemis found that poor functional results tended to occur in those cases delayed for 1 year or longer, 5 although this is not the experience of all authors. ${ }^{6,9}$ Similarly, the age of the patient has been found
Figure 6. Distal mastoid segment of case 16. Light microscopy shows marked fibrosis with only a few scattered myelinated axons. (Toluidine blue $\times 210$.)

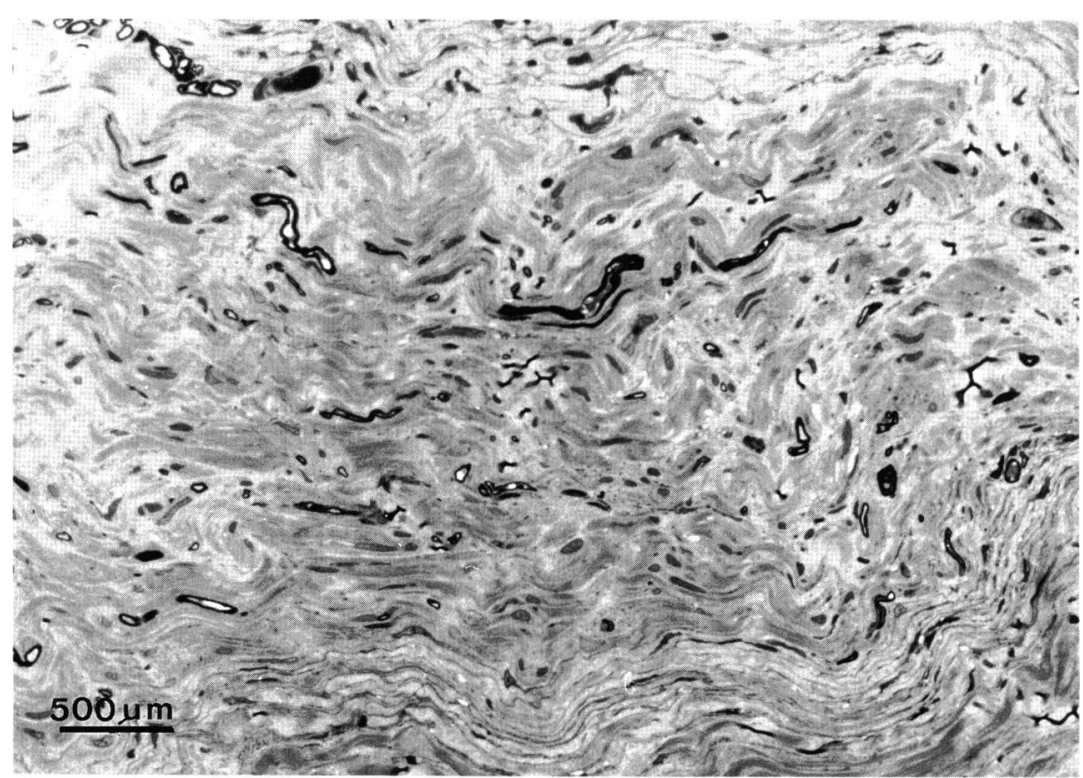




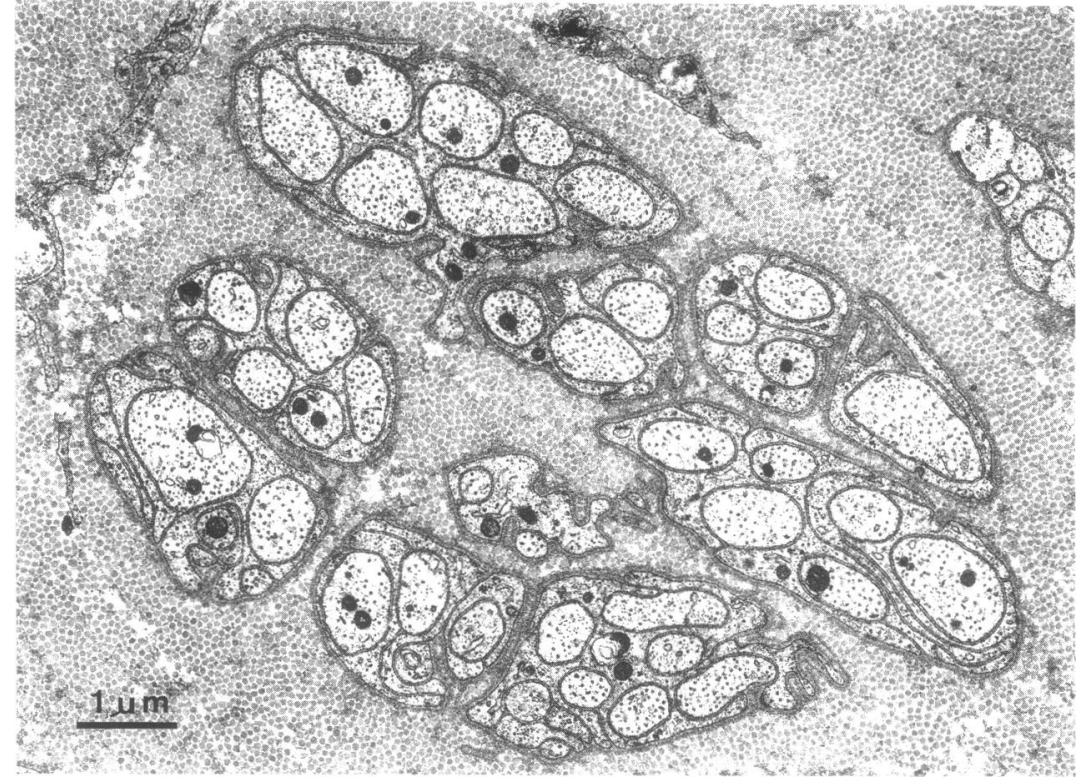

Figure 7. Electron micrograph of case 16. Endoneural tubes containing nonmyelinated axons are seen between the densely packed collagen fibrils. (Uranyl acetate and lead citrate $\times 10200$.) to be significant in some studies, with poorer results in older patients. ${ }^{5,6}$ In the group of patients studied, there was no evidence of a relationship between functional results and either duration of palsy prior to anastomosis or age of the patients. Twelve of the patients had their anastomosis 2 years or more after the facial paralysis, with one anastomosis after a delay of 7 years. It is interesting to note that there is no significant difference between the two groups. However, these results should be qualified by the consideration that the patients selected for the procedure had at least some degree of preoperative facial tone. The finding of a sensory bundle in the 6 cases in which the lesion was proximal to the geniculate ganglion is consistent with Wallerian law. The origin of a bundle of intact myelinated small-diameter fibers in a glomus tumor that had infiltrated the nerve distal to the geniculate ganglion is unclear. Possibly these fibers originate from the auricular branch of the vagus. There was evidence of ongoing axon degeneration in palsies of up to 72 months duration. It is unclear whether the degenerating fibers are surviving ones from the initial injury or are regenerated myelinated fibers. Evidence of active axon degeneration was significantly related to the outcome of surgery. The mean postoperative result in the group of patients without axon degeneration was $61 \%$, compared to $50 \%$ in those with degeneration. This level of difference may be large enough to be clinically as well as statistically significant. It is possible that the cellular changes associated with the axon degeneration are inhibitory to ingrowing axons. The finding of pronounced phagocytic activity in the case in which the nerve had been sectioned 1 month before suggests that the initial degenerative changes in the human facial nerve may proceed at a slower tempo than indicated by animal studies. This finding is consistent with the results of Ylikoski et al. 4

The nature of the injury in these patients is hetero- genous. However, with the exception of case number 3 , where the nerve had been transected, all the cases would be expected to be axonotmeses, with distal loss of axons and their myelin sheaths. ${ }^{10}$ Endoneural tubes with nonmyelinated axons were a universal finding. Remarkably, they were present in two cases in which there was a break in continuity of the nerve. This observation has been made previously in one case where the nerve had been transected $^{4}$ and distal to fibrosed segments following temporal bone fractures. ${ }^{11}$ The origin of these fibers is still not clear. While it is generally held that these fibers are regenerating motor axons, their relationship to the small myelinated fibers seen in some of the cases is unknown. It is possible that these fibers remain nonmyelinated until they make contact with a motor endplate or, alternatively, that they are myelinated fibers that have lost their myelin. Their presence in the early cases and their apparent persistence argue against this latter possibility - it is interesting to note the tendency for endoneural tubes as well as myelinated axons to increase with the duration of the palsy. The presence of endoneural tubes might be expected to provide favorable conditions for regeneration after anastomosis, and indeed, in these patients there did appear to be a relationship between their relative numbers and the postoperative facial function. There is evidence to suggest that the morphology of the facial muscle fibers may be important in determining graft outcome. ${ }^{12}$ It is interesting to speculate that the nonmyelinated fibers relate to a favorable outcome because in some way they maintain the motor endplates. The relative numbers of myelinated axons, on the other had, showed no association with the postoperative results.

The occurrence of intraneural fibrosis would be expected to impede the ingrowth of axons. Experiments in which peripheral nerves are sectioned in animals result in fibrosis within weeks. ${ }^{1}$ This suggests that the sooner anas- 
tomosis is carried out, the greater the chances of success. In relation to this, two features stand out in this study. First, although there was a general increase in endoneural collagen in all cases after 1 month, there was no tendency for collagenosis to increase with the duration of facial palsy. Second, fibrosis was not a frequent feature: extensive fibrosis occurred only in one case, which was an idiopathic palsy of 24 months duration. It is interesting to note that despite the fibrosis the nerve showed an extensive ingrowth of nonmyelinated axons, although the significance of this finding is unclear. The functional result in this case was $50 \%$ after 4 years follow-up, and it is therefore clear that even where fibrosis occurs it does not necessarily prevent the ingrowth of axons or preclude some return of function.

\section{CONCLUSIONS}

There is no histological evidence of increasing collagenosis or fibrosis with duration of facial palsy, endoneural fibrosis being a very infrequent finding. There is no evidence that the relative numbers of endoneural fibrocytes or the degree of collagenosis is related to the functional result. The presence of large numbers of endoneural tubes, filled with nonmyelinated axons, may indicate a favorable prognosis. In contrast, the presence of active axon degeneration in the facial nerve may indicate a poor prognosis. Provided that there is some degree of preoperative facial muscle tone, there is no evidence that a delay in anastomosis beyond 2 years adversely affects the postoperative return of facial function. The histological condi- tion of the hypoglossal nerve may be more important than the condition of the facial and should be examined by frozen section at the time of anastomosis.

\section{REFERENCES}

1. Sunderland S, Bradley KC: Denervation atrophy of the distal stump of a severed nerve. J Comp Neurol 93:401-409, 1950

2. Sunderland S: Nerves and Nerve Injuries. 2nd ed. Edinburgh: Churchill Livingston, 1978

3. Ylikoski J, Brackmann DE, Savolainen S: Facial nerve abnormalities after acoustic tumor removal. Morphological and clinical study of seven patients with postoperative facial paralysis. Arch Otolaryngol 108:795-800, 1982

4. Ylikoski J, Hitselberger WE, House WF, Sanna M: Degenerative changes in the distal stump of the severed human facial nerve. Acta Otolaryngol 92:239-248, 1981

5. Gavron JP, Clemis JD: Hypoglossal-facial nerve anastomosis: A review of forty cases caused by facial nerve injuries in the posterior fossa. Laryngoscope 94:1447-1450, 1984

6. Sabin HI, Bordi LT, Symon L, Compton JS: Facio-hypoglossal anastomosis for the treatment of facial palsy after acoustic neuroma resection. $\mathrm{Br} \mathrm{J}$ Neurosurg 4:313-317, 1990

7. Conley J, Baker DC: Hypoglossal-facial anastomosis for reinnervation of the paralysed face. Plast Reconstr Surg 63:63-72, 1979

8. Fisch U: Surgery for Bell's palsy. Arch Otolaryngol 107:1-11, 1981

9. Hitselberger WE: Hypoglossal-facial anastomosis. Otolaryngol Clin North Am 7:545-550, 1974

10. Seddon HJ: Three types of nerve injury. Brain 66:237, 1943

11. Felix H, Eby TL, Fisch U: New aspects of facial nerve pathology in temporal bone fractures. Acta Otolaryngol 111:332-336, 1991

12. Frey M, Happak W, Girsch W, Bittner RE, Gruber H: Histomorphometric studies in patients with facial palsy treated by functional muscle transplantation: New aspects for the surgical concept. Ann Plast Surg 26:370-379, 1991

We are most grateful to Mrs. V. Hoffmann and Mrs. G. Gantenbein for their excellent technical assistance. 